\title{
Detection of Impact Location by Using Anisotropy of Output Voltage of Metal-Core Piezoelectric Fiber/Aluminum Composites
}

\author{
Tetsuro Yanaseko ${ }^{1,}$, , Hiroshi Asanuma ${ }^{2}$, Hiroshi Sato ${ }^{3}$ \\ ${ }^{1}$ Division of Material Science and Engineering, Yokohama National University, Yokohama, Japan \\ ${ }^{2}$ Department of Mechanical Engineering, Chiba University, Chiba, Japan \\ ${ }^{3}$ National Institute of Advanced Industrial Science and Technology, Tsukuba, Japan \\ Email address: \\ yanaseko-tetsurou@ynu.ac.jp (T. Yanaseko), asanuma@faculty.chiba-u.jp (H. Asanuma), h-sato@aist.go.jp (H. Sato)
}

\section{To cite this article:}

Tetsuro Yanaseko, Hiroshi Asanuma, Hiroshi Sato. Detection of Impact Location by Using Anisotropy of Output Voltage of Metal-Core Piezoelectric Fiber/Aluminum Composites. International Journal of Materials Science and Applications. Vol. 4, No. 4, 2015 , pp. 256-260. doi: 10.11648/j.ijmsa.20150404.15

\begin{abstract}
This paper describes the detection of an impact location by using anisotropy of the output voltage of a metal-core piezoelectric ceramic fiber/aluminum composite. Metal-core piezoelectric ceramic fibers are very fragile. The mechanical properties of metal-core piezoelectric ceramic fibers can be improved by embedding the fibers into the metals that have excellent reliability and strength. Therefore, metal-core piezoelectric ceramic fiber/aluminum composites were developed by using the Interphase Forming/Bonding method. Furthermore, the output voltage characteristics of fabricated a composite were evaluated, and the anisotropy of the output voltage was observed. In this study, the impact location detection system was developed by using the anisotropy of the output voltage of the composite. It is expected to reduce the number of sensors compared with conventional systems while maintaining the accuracy. As a result, the impact location detection was successfully developed by using the model of the output voltage of the composites that were disposed in two different positions to obtain the signals.
\end{abstract}

Keywords: Structural Health Monitoring, Smart Material, Piezoelectric Material, Sensor, Aluminum, Metal Matrix Composite

\section{Introduction}

In recent years, there has been a great deal of interest in structural health monitoring (SHM) technology, especially as it applies to the maintenance and management of infrastructures [1-3]. In SHM, sensors which using piezoelectric ceramics are often used [4-6]. Piezoelectric ceramics are widely used in sensors and actuators because of excellent responsibility, sensitivity and high generated force. On the other hand, the applicable scope of the piezoelectric ceramics is limited because of their fragility. To overcome these problems, Asanuma et al. embedded platinum-core piezoelectric ceramic fibers [7-10] in aluminum using the Interphase Forming/Bonding (IF/B) method [11] and fabricated a composite without causing mechanical or electrical damage to the piezoelectric fiber [12-16]. Furthermore, the output voltage characteristics of the fabricated composite were evaluated, and the anisotropy of the output voltage was observed [17]. In the conventional SHM system, the sensors are arranged to surround the measurement area [18-19], however this arrangement has led an increase in the number of the sensors. Therefore, an impact detection system that can reduce the number of sensors while ensuring the measurement accuracy was developed by utilizing the output voltage anisotropy.

\section{Experimental}

\subsection{Materials}

The materials used in this study are summarized in Table 1. Pure aluminum plates were used as a matrix and backing plate, and a pure copper foil was used as an insert material for the eutectic reaction and an electrode. The metal-core 
piezoelectric fiber was made of lead zirconate titanate (PZT), which has a platinum core as a functional fiber.

Table 1. Cross section of the materials prepared for hot pressing.

\begin{tabular}{llll}
\hline Material & Young's modulus, E/GPa & Dimmension/mm & Usage \\
\hline Aluminum & 70.6 & $l=20, w=30, t=0.2,0.4,1.2$ & Matrix, Bucking plate \\
Metal-core piezoelectric fiber & 120 & Diameter: 0.2 Length: 25 & Functional fiber \\
Copper & - & $1=20, \mathrm{w}=30, \mathrm{t}=0.01$ & Insert \\
& & $l=3, w=6, t=0.02$ & Electrode \\
\hline
\end{tabular}

\subsection{Fabrication of Metal-Core Piezoelectric Fiber /Aluminum Composites}

For fabricating the specimens, $0.2,0.4$ and $1.2 \mathrm{~mm}$ thick pure aluminum plates and $0.01 \mathrm{~mm}$ thick copper foil were cut in the dimensions of $15 \times 30 \mathrm{~mm}$ and polished with waterproof abrasive paper to remove the oxide film. The 0.2 and the $1.2 \mathrm{~mm}$ thick aluminum plates were bonded using a cyanoacrylate adhesive. The copper foil was then placed on the bonded plates and a $0.25 \mathrm{~mm}$ SUS304 stainless steel wire was centered in the lengthwise direction and pressed in the middle by means of a pressing machine to form a U-groove. After placing a metal-core piezoelectric fiber of length $20 \mathrm{~mm}$ in the groove, the $0.2 \mathrm{~mm}$ thick aluminum plate was placed on top and hot pressed. Figure 1 shows the laminate configuration of the specimen. The aluminum plates and copper foil were hot-pressed at the temperature of $873 \mathrm{~K}$, at the pressure of $2.2 \mathrm{MPa}$ and for the period of $2.4 \mathrm{ks}$ and in a vacuum of $0.1 \mathrm{kPa}$. After hot pressing, the composites were cut into square-shaped test pieces of dimension $20 \times 20 \mathrm{~mm}$, and removed from the backing plate. Electrodes were attached to the composites pieces to form patch type specimens as shown in Fig. 2. The piezoelectric fiber was polarized by applying $300 \mathrm{~V}$ between the aluminum matrix and platinum core for $1.8 \mathrm{ks}$.

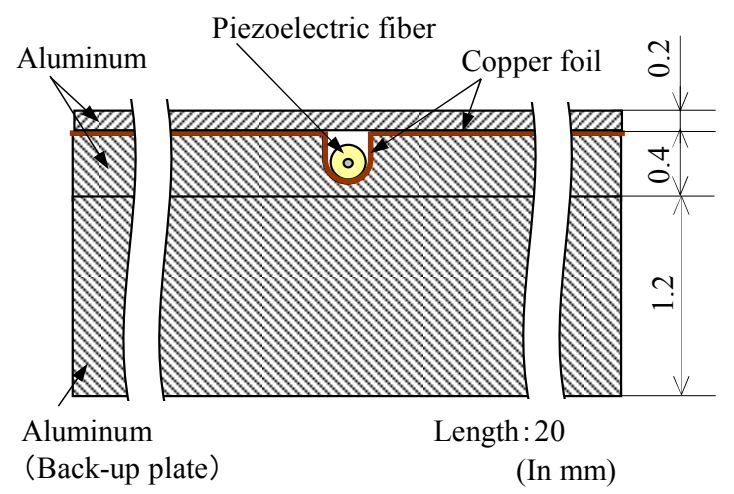

Figure 1. Cross section of the materials prepared for hot pressing.

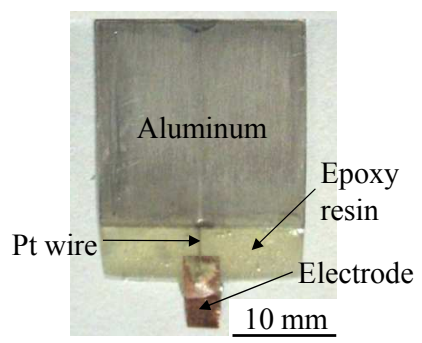

Thickness : $0.55 \mathrm{~mm}$

Figure 2. General appearance of specimen.

\subsection{Formulation of Output Voltage}

The output voltage characteristics of the fabricated specimens were evaluated by the impact test, and the formulation of the output voltage was carried out from the obtained results. The output voltage was formulated using three parameters; distance attenuation, the anisotropy of output voltage and the impact energy dependence. Figure 3 shows the impact test system to evaluate output voltage characteristics. Specimens A and B were fixed on a duralumin plate (A2017P-T351) using a cyanoacrylate adhesive as shown in the figure.

For the evaluation of the distance attenuation, the impact was applied by dropping a steel ball (mass: $3.5 \mathrm{~g}$ ) from the falling height $\mathrm{h}=300 \mathrm{~mm}$, and the impact distance $\mathrm{d}$ between the impact point and the centers of specimens A and B was increased from $25 \mathrm{~mm}$ to $200 \mathrm{~mm}$ in steps of $25 \mathrm{~mm}$. The distance attenuation was formulated from the obtained output voltage.

To examine the effect of the impact energy on the output voltage and the anisotropy of the output voltage, the falling height $\mathrm{h}$ was increased from 50 to $300 \mathrm{~mm}$ in $50 \mathrm{~mm}$ increments, and the output voltage generated because of the impact was measured. In this test, the impact points were 50 $\mathrm{mm}$ from the centers of the specimens in $\mathrm{x}$-direction and 50 $\mathrm{mm}$ from the centers of the specimens in y-direction.

In this study, the origin of the $x-y$ coordinate was set at the center of specimen $\mathrm{A}$, and the fiber direction of specimens was confirmed to y-direction.

From the formulated characteristics, the distance attenuation, impact energy dependence and the anisotropy of the output voltage, the impact position was estimated. Figure 4 shows the points of the impact in this study. The procedure is explained as follows:

1) Impact is applied to a point within the defined area, and the coordinates of the impact point is set to (xi, yi).

2) Predicted trajectory is calculated by Eq. (1) from the propagation velocity of the surface wave $\mathrm{v}$ and the difference of the rise time of the output voltage waveform $t$ obtained from the specimens A and B.

$$
\sqrt{\left(x_{i}-x_{A}\right)^{2}+\left(y_{i}-y_{A}\right)^{2}}-\sqrt{\left(x_{i}-x_{B}\right)^{2}+\left(y_{i}-y_{B}\right)^{2}}=v \Delta t
$$

where, $(\mathrm{xA}, \mathrm{yA})$ and $(\mathrm{xB}, \mathrm{yB})$ are the coordinates of the centers of specimens $\mathrm{A}$ and $\mathrm{B}$. The measured value of the propagation velocity of surface wave is $2710 \mathrm{~m} / \mathrm{s}$.

3) The impact energy is estimated by using the formulated characteristics at each point on the predicted trajectory. The estimated impact location is the point where the difference 
between estimated impact energy obtained from the output voltage of specimens A and B is minimum.

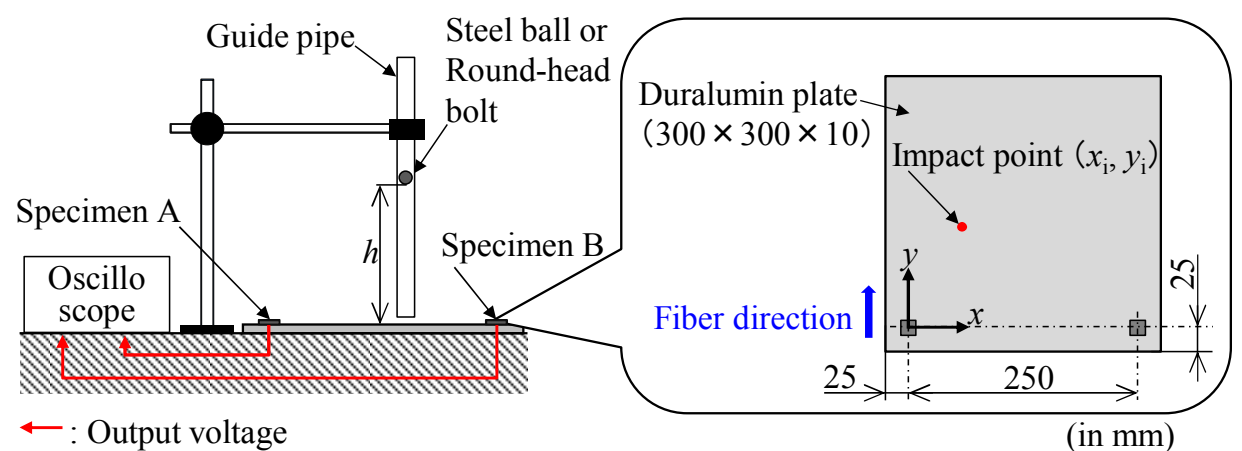

Figure 3. Impact detection test system.

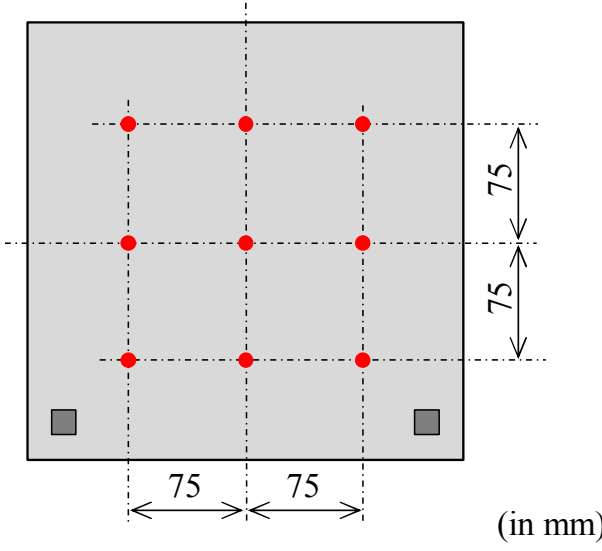

Figure 4. Impact point to detect of impact location.

\section{Results and Discussions}

\subsection{Formulation of Output Voltage}

\subsubsection{Distance Attenuation of Output Voltage}

The vibration wave front enlarges and the vibration amplitude decreases with transmission, which is referred to as geometric attenuation. The geometric attenuation can be expressed by Eq. (2) as below.

$$
\Delta V=20 \log \left(\frac{d}{d_{b}}\right) g_{a}
$$

where,

V: Voltage attenuation

d: Distance between the impact point and the specimen

$\mathrm{db}$ : Reference distance

ga: Geometrical attenuation factor

Here, the reference distance $\mathrm{db}$ is $25 \mathrm{~mm}$, and the geometric attenuation factor is 0.5 because the surface waves are used to estimate the impact point in this experiment. The experimental results to evaluate the distance attenuation of the output voltage and calculated values according to Eq. (2) are shown as Fig. 5. The calculated and experimental values were found to be in good agreement, and the distance between the impact point and the specimen could be estimated from Eq. (2).

\subsubsection{Impact Energy Dependence and Anisotropy of Output Voltage}

Figure 6 shows the results of the impact energy dependence of the output voltage evaluation test. According to this figure, it can be seen that the output voltage increases almost proportionately with the square root of the impact energy. This elastic energy, which is that converted from the impact energy is proportional to the square of the strain, and the output voltage is linear to the strain. Thus, the output voltage is proportional to the square root of the impact energy.

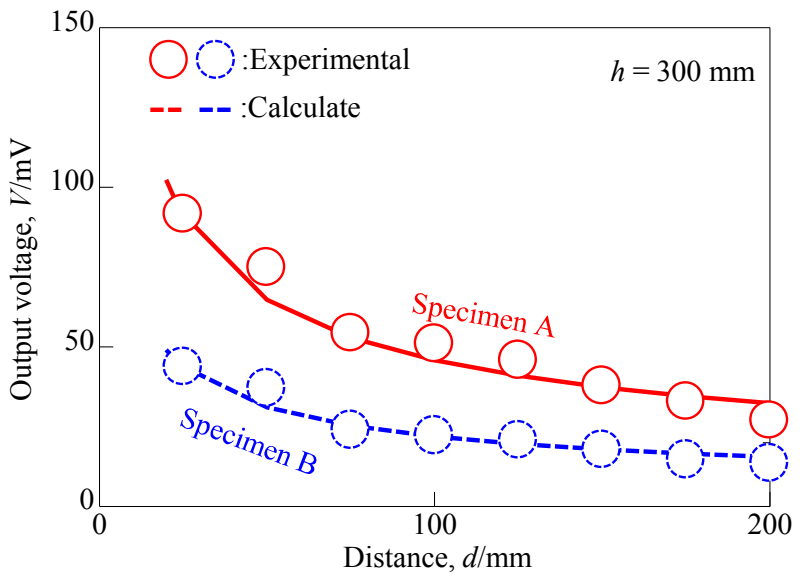

Figure 5. Effect of the distance on the output voltage.

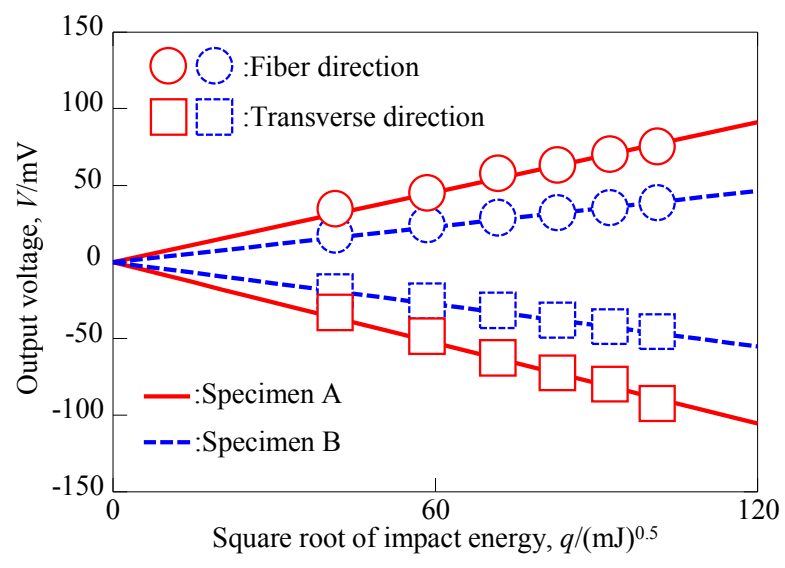

Figure 6. Effect of the impact energy on the output voltage. 
Moreover, the output voltage sign reversed on changing the direction of the impact from $0^{\circ}$ to $90^{\circ}$. These results show that this composite has anisotropy of sensitivity. The output voltage due to the strain in the fiber direction is dependent on the piezoelectric constant $\mathrm{d} 31$. However, the output voltage due to the strain in the transverse direction of the fiber is dependent on the piezoelectric constants $\mathrm{d} 33$ and $\mathrm{d} 31$. In the case of general piezoelectric materials represented by PZT, $\mathrm{d} 31$ and $\mathrm{d} 33$ have opposite signs and the absolute value of the $\mathrm{d} 33$ constant exceeds that of the $\mathrm{d} 31$. Because of this, in the $90^{\circ}$ direction, $\mathrm{d} 33$ becomes predominant, which may cause the reversal of the waveform.

In addition, the anisotropy of the output voltage is represented by Eq. (3).

$$
V=D_{x} \varepsilon \frac{1-\cos 2 \alpha}{2}+D_{y} \varepsilon \frac{1+\cos 2 \alpha}{2}
$$

where, Dx and Dy are coefficients of the output voltage in $\mathrm{x}$ -direction and y-direction, and is the strain direction. The output voltage anisotropy was calculated using the results of this experiment, as shown in Fig. 7. According to the figure, it was found that the output voltage become $0 \mathrm{~V}$ at around $45^{\circ}$, because the fiber direction output voltage and the fiber transverse direction output voltage canceled out each other.

\subsection{Estimation of Impact Location}

The impact point was estimated based on the formulated characteristics obtained above. The difference between the estimated results and the points that actually applied impact are shown in Fig. 8. The maximum estimation error was about $9 \mathrm{~mm}$. Furthermore, from the estimation results, it can be seen that the error increases at the impact points on the diagonal of the impact point group that shown in Fig. 4. This increase in the estimation error is due to the anisotropy of the output voltage as described in Eq. (3). Because the point on the diagonal line is around the direction that the phase of the output voltage switches, therefore the output voltage rapidly reduces and the measurement error becomes relatively large. Moreover, to improve the estimation accuracy, it is necessary to produce the anisotropy of the output voltage and ensure the output voltage in the vicinity of the diagonal. This is achieveed by adjusting the anisotropy of the output voltage by the design of the electrode structure of the composite.

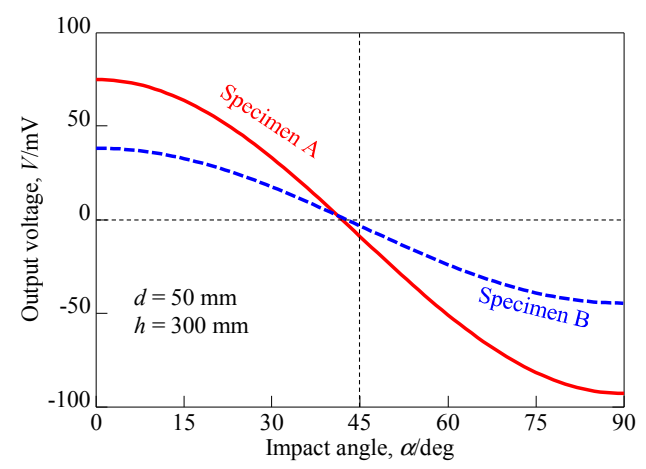

Figure 7. Calculation results of the anisotropy of the output voltage.

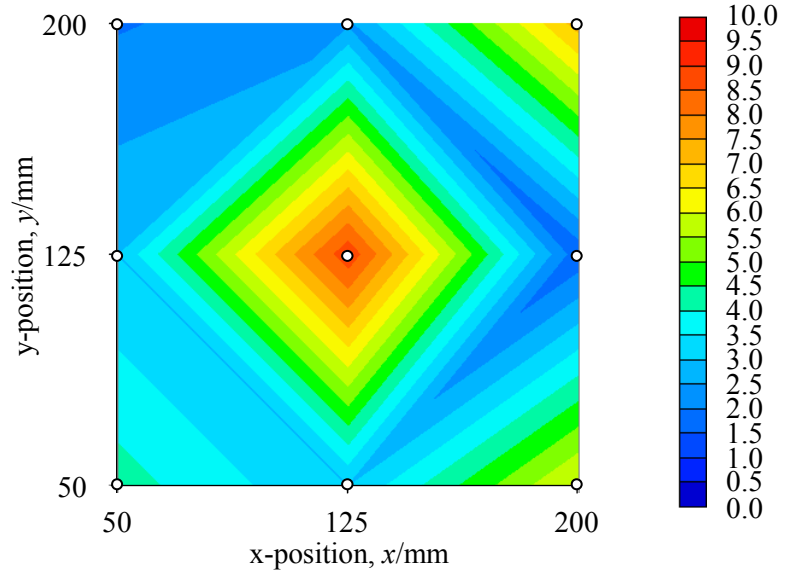

Figure 8. Error between estimated result and impact point.

\section{Conclusions}

In this study, the impact detection using the output voltage anisotropy of the piezoelectric fiber/aluminum composite was investigated, and the conclusions were obtained as below.

1) The distance attenuation of the output voltage of the piezoelectric fiber/aluminum composite can be represented by the geometric attenuation model.

2) The output voltage is proportional to the square root of the impact energy, and the voltage varies depending on the strain direction.

3) By using the anisotropy of the output voltage, it is possible to estimate the impact location, and the maximum estimation error is $8.88 \mathrm{~mm}$.

4) The maximum estimation error occurred by an increase in the measurement error due to rapid decrease in the output voltage caused the anisotropy of the output voltage. Therefore, the accuracy is expected to improve by adjusting the anisotropy.

\section{Acknowledgements}

This study was supported by jsps kakenhi Grant Number 24360043. Authors are grateful for their support.

\section{References}

[1] J. M. W. Brownjohn, "Structural health monitoring of civil infrastructure," Philosophical Transactions of the Royal Society A, 365 (2007), pp 589-622.

[2] E. Aktan, A. J. Helmicki, V. J. Hunt, "Issues in health monitoring for intelligent infrastructure," Smart Materials and Structures, 7 (1998), pp. 674-692.

[3] E. Aktan, F. N. Catbas, K. A. Grimmelsman, C. J. Tsikos, "Issues in infrastructure health monitoring for management," Journal of Engineering Mechanics, ASCE, 126 (2000), pp. $711-724$.

[4] H. Fukunaga, N. Hu, F-K. Chang, "Structural damage identification using piezoelectric sensors," International Journal of Solids and Structures, 39 (2002), pp. 393-418. 
[5] W. H. Duan, Q. Wang, S. T. Quek, Applications of Piezoelectric Materials in Structural Health Monitoring and Repair: Selected Research Examples, Materials, 3 (2010), pp. 5169-5194.

[6] S. S. Kessler, S. M. Spearing, "Design of a piezoelectric-based structural health monitoring system for damage detection in composite material," Proceedings of SPIE, 86 (2005), Paper No. 4701

[7] H. Sato, Y. Shimojo, and T. Sekiya, "Lead zirconate titanate fiber, smart board using lead zirconate titanate fiber, actuator utilizing smart board, and sensor utilizing smart board," US patent, US6963157 B2 (2005).

[8] J. Qiu, J. Tani, N. Yamada, and H. Takahashi, "Fabrication of piezoelectric fibers with metal core," Proceedings of. SPIE, Smart Structures and Materials 2003: Active Materials: Behavior and Mechanics (2003), Paper No. 5053.

[9] H. Sato, T. Sekiya and M. Nagamine, "Design of the metal-core piezoelectric fiber," Proceedings of SPIE, Smart Structures and Materials 2004: Smart Structures and Integrated Systems (2004), Paper No. 5390.

[10] H. Sato and M. Nagamine, "Mechanical properties of metal-core piezoelectric fiber," Proceedings of SPIE, Smart Structures and Materials 2005: Smart Structures and Integrated Systems (2005), Paper No. 5764.

[11] H. Asanuma, "Development of metal-based smart composites," JOM, Vol. 52, No. 10 (2000), pp. 21-25.

[12] H. Asanuma, N. Takeda, T. Chiba and H. Sato, "Fabrication of metal core piezoelectric fiber/aluminum composite material," Nippon Kikai Gakkai (The Japan Society of Mechanical Engineers), Collection of papers of 14th Mechanical material and material processing technology lecture meeting (2006), pp. 21-22.
[13] H. Asanuma and H. Sato, "Functional composite material equipped with embedded piezoelectric fiber with metal core," Chiba University, National Institute of Advanced Industrial Science and Technology, Japanese Patent No. 4719897.

[14] D. Askari, R. Ruth, H. Asanuma and M. N. Gasemi-Nejhad, “A comparative study on macrofiber composites and active fiber composites with metal-core piezoelectric actuators/sensors," Proceedings of SPIE, Smart Structures and Materials 2006: Active Materials: Behavior and Mechanics (2006), Paper No. 6170 .

[15] H. Asanuma, J. Kunikata and M. Kibe, "Development of multifunctional structural material systems by innovative design and processing," Materials Research Society Symposium Proceedings, Vol. 1129 (2009), pp. 251-262.

[16] M. Richeson, U. Erturun, R. Waxman, K. Mossi, J. Kunikata and $\mathrm{H}$. Asanuma, "Characterization of a Pt-core PZT fiber/Al matrix composite", Proceedings of SPIE, Behavior and Mechanics of Multifunctional Materials and Composites 2010 (2010), Paper No. 7644.

[17] T. Yanaseko, H. Asanuma, H. Sato, "Characterization of a metal-core piezoelectric ceramics fiber/aluminum composite," Mechanical Engineering Journal, 2, 2 (2015), pp. 1-8.

[18] X. Zhao, H. Gao, G. Zhang, B. Ayhan, Y. Yan, C. Kwan, J. L. Rose, "Active health monitoring of an aircraft wing with embedded piezoelectric sensor/actuator network: I. Defect detection, localization and growth monitoring," Smart Materials and Structures, 16 (2007), pp. 1208-1217.

[19] M. Lehmann, A. Büter, B. Frankenstein, F. Schubert, B. Brunner, "Monitoring System for Delamination Detection Qualification of Structural Health Monitoring (SHM) Systems," Conference on Damage in Composite Material CDCM 2006, Stuttgart, September 2006. 